\title{
MOTION OF A VISCOUS FLUID IN A TUBE WHICH IS SUBJECTED TO A SERIES OF PULSES*
}

\section{By G. N. LANCE (Department of Mathematics, Cniversity of Southampton, England)}

Introduction. Consider a circular pipe which contains a viscous fluid. The flow due to a constant pressure gradient in a stationary pipe is the well-known Poiseuille flow. Now suppose that a pipe, containing a viscous fluid, is subjected to a series of pulses in an axial direction which do not appreciably move the pipe. The fluid inside will acquire a velocity in the direction of the pulses, relative to the pipe, due to the transfer of momentum from the boundary by viscous stresses. We determine the flow, relative to the pipe, which is obtained when a pressure gradient and a series of pulses act. Since the equation of motion is linear the two effects may be considered separately and the two results added together.

In particular we are interested in the case when the pressure gradient and the pulses have opposite senses. In this case there arises the possibility that the discharge from the pipe could be completely stopped.** In Sec. 1 we consider the simpler case of flow in a two dimensional channel. The case of a pipe with circular cross-section is taken up in Sec. 3.

1. Flow in a straight walled two-dimensional channel. Let $x$ be the coordinate measured along the channel length, $2 a$ be the distance between the walls and the origin of coordinates be midway between the walls.

For the steady state motion the velocity in the axial direction $u(y)$ satisfies the differential equation

$$
\mu \frac{d^{2} u}{d y^{2}}=\frac{d p}{d x}
$$

If the pressure gradient $d p / d x=-\rho P$ (a constant) then the velocity is given by the expression

$$
u(y)=\frac{P a^{2}}{2 \nu}\left[1-\left(\frac{y}{a}\right)^{2}\right],
$$

where the viscous boundary condition of zero slip on the planes $y= \pm a$ has been used. The result may be rewritten

$$
u(y)=\frac{3 u_{m}}{2}\left[1-\left(\frac{y}{a}\right)^{2}\right],
$$

where $u_{m}=P a^{2} / 3 \nu$ is the mean value of $u$ across the width of the channel.

The time dependent equation is

$$
\mu \frac{\partial^{2} u}{\partial y^{2}}-\rho \frac{\partial u}{\partial t}-\rho P=0 .
$$

${ }^{*}$ Received March 24, 1955; revised manuscript received August 16, 1955. This paper was written when the author was in the Department of Engineering, University of California, Los Angeles, and he wishes to acknowledge receipt of a maintenance grant of the F. O. A., Scientific Research Project TA01-101-3006 (OEEC 151).

**This problem was suggested by reports that the engines of certain jet aircraft fail when the guns are fired. The failure could be due to fuel starvation. 
The flow velocity $u(y, t)$ due to the pressure gradient and the action of pulses can be considered (since the equation is linear) to be composed of the flow (2) and an additional velocity $u_{1}(y, t)$. Introducing non-dimensional variables $v, \xi$ and $T$ defined by

$$
\begin{aligned}
u_{1}(y, t) & =u_{m} v(\xi, T), \\
y & =a \xi,
\end{aligned}
$$

and

$$
t=a^{2} T / \nu,
$$

the equation for $v(\xi, T)$ is

$$
\frac{\partial^{2} v}{\partial \xi^{2}}=\frac{\partial v}{\partial T}
$$

2. Boundary conditions. The boundary and initial conditions which must be imposed on $v$ are

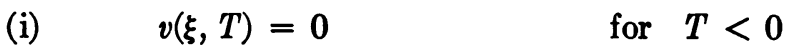

$$
\begin{aligned}
& \text { (ii) } \quad v( \pm 1, T)=\sum_{0}^{N} A_{n} \delta(T-n D) \text { for } T \geq 0 \text {. }
\end{aligned}
$$

In (ii) $A_{n}$ is a non-dimensional quantity giving the strength of the $n$th pulse and $D$ is the time interval between pulses. The differential equation (8) can be solved, subject to the conditions (9) and (10), by the Laplace transform technique. If capital letters denote the Laplace transform, with respect to $T$, of the corresponding small letters then we get

$$
\frac{d^{2} V}{d \xi^{2}}=p V
$$

and

$$
V( \pm 1, p)=\sum_{0}^{N} A_{n} e^{-p n D}
$$

Thus

$$
V(\xi, p)=\frac{\cosh \xi p^{1 / 2}}{\cosh p^{1 / 2}} \sum_{0}^{N} A_{n} e^{-p n D} .
$$

The transform of the quantity of fluid flowing (relative to the channel) per unit width of channel, per unit time, is

$$
Q(p)=\int_{-1}^{+1} V(\xi, p) d \xi=2 p^{-1 / 2} \tanh p^{1 / 2} \sum_{0}^{N} A_{n} e^{-p n D} .
$$

The inverse transform of (14) can be found in many tables. (See for example B. van der Pol and H. Bremmer [1].) We find

$$
q(T)=2 \sum_{0}^{N} A_{n} \Theta_{2}(0,[T-n D] / \pi) H([T-n D] / \pi),
$$

where $H(x)$ is the unit function. 
The theta function which enters may be expressed in the form

so that

$$
\Theta_{2}(0, t)=2 \sum_{0}^{\infty} \exp \left[-\pi\left(m+\frac{1}{2}\right)^{2} t\right]
$$

$$
q(T)=4 \sum_{n=0}^{N} A_{n} H([T-n D] / \pi) \sum_{m=0}^{\infty} \exp \left[-\left(m+\frac{1}{2}\right)^{2}(T-n D)\right] .
$$

3. Flow in a pipe of circular cross section. We turn now to the case of axially symmetric flow in a pipe of circular cross section. The radial coordinate is $r$ and the axial direction is $x$ as before. The treatment follows exactly that used in Secs. 1 and 2. In the present case we require a solution of the differential equation

$$
\frac{\partial^{2} v}{\partial \xi^{2}}+\frac{1}{\xi} \frac{\partial v}{\partial \xi}=\frac{\partial v}{\partial T}
$$

where $r=a \xi, a$ is the radius of the pipe and $u_{m}=P a^{2} / 8 \nu$ in the present case. The boundary conditions are

$$
\begin{aligned}
& \text { (i) } \quad v(\xi, T)=0 \text { for } T<0, \\
& \text { (ii) } \quad v(1, T)=\sum_{0}^{N} A_{n} \delta(T-n D),
\end{aligned}
$$

with the further condition that $v$ is finite on the axis.

The Laplace transform $V(\xi, p)$ of $v(\xi, T)$ satisfies

subject to

$$
\frac{d^{2} V}{d \xi^{2}}+\frac{1}{\xi} \frac{d V}{d \xi}=p V
$$

Hence

$$
V(1, p)=\sum_{0}^{N} A_{n} e^{-p n D} \text {. }
$$

$$
V(\xi, p)=\frac{J_{0}\left(i \xi p^{1 / 2}\right)}{J_{0}\left(i p^{1 / 2}\right)} \sum_{0}^{N} A_{n} e^{-p n D} .
$$

The transform of the quantity of fluid flowing (relative to the channel) per unit time is

$$
Q(p)=2 \pi \int_{0}^{1} \xi V(\xi, p) d \xi=\frac{2 \pi}{i p^{1 / 2}} \frac{J_{1}\left(i p^{1 / 2}\right)}{J_{0}\left(i p^{1 / 2}\right)} \sum_{0}^{N} A_{n} e^{-p n D} .
$$

The inverse transform of $Q(p)$ is

$$
q(T)=2 \pi \frac{\partial}{\partial T}\left\{\sum_{0}^{N} A_{n}\left(\frac{1}{2}-2 \sum_{m=1}^{\infty} \alpha_{m}^{-2} \exp \left[-\alpha_{m}^{2}(T-n D)\right]\right) H(T-n D)\right\}
$$

or

$$
\begin{aligned}
q(T)=2 \pi \sum_{0}^{N} A_{n}\left\{2 \sum_{m=1}^{\infty}\right. & \exp \left[-\alpha_{m}^{2}(T-n D)\right] H(T-n D) \\
+ & {\left.\left[\frac{1}{2}-2 \sum_{m=1}^{\infty} \alpha_{m}^{-2} \exp \left\{-\alpha_{m}^{2}(T-n D)\right\}\right] \delta(T-n D)\right\}, }
\end{aligned}
$$

where $\alpha_{m}$ is the $m$ th root of the Bessel function $J_{0}(x)$. 
4. Discussion of the results. In the case of the two dimensional channel the basic Poiseuille flow causes a discharge obtained from (3) of amount $2 a u_{m} /$ unit width of channel. Thus if we confine ourselves to one pulse the total discharge at time $T$ is

$$
a u_{m}\left\{2+4 A_{0} H(T / \pi) \sum_{m=0}^{\infty} \exp \left[-\left(m+\frac{1}{2}\right)^{2} T\right]\right\} \text {. }
$$

Since the pulses act in a direction opposing the free streaming $A_{0}$ is negative and if $T$ is such that

$$
\frac{1}{2}<-A_{0} \sum_{m=0}^{\infty} \exp \left[-\left(m+\frac{1}{2}\right)^{2} T\right]
$$

then the flow will be reversed. This inequality can clearly be satisfied if $T$ is small. The length of time for which the flow is arrested will depend on the magnitude of the impulse coefficient $A_{0}$. Alternatively, if another impulse follows the first rapidly enough, the flow will remain arrested for a further period.

In the case of the circular pipe the basic flow causes a discharge $\pi a^{2} u_{m}$. So the total flux due to a single pulse and the basic flow is

$$
a^{2} u_{m}\left(\pi+2 \pi A_{0}\left\{2 \sum_{m=1}^{\infty} \exp \left(-\alpha_{m}^{2} T\right) H(T)+\left[\frac{1}{2}-2 \sum_{m=1}^{\infty} \alpha_{m}^{-2} \exp \left(-\alpha_{m}^{2} T\right)\right] \delta(T)\right\}\right) .
$$

It follows, as before that when $T$ is small but not zero the flow will be arrested if

$$
\frac{1}{4}<-A_{0} \sum_{m=1}^{\infty} \exp \left(-\alpha_{m}^{2} T\right) .
$$

Conclusions. The above analysis shows that it is entirely possible for the flow of a viscous fluid, in a pipe or channel, to be arrested when the pipe is subjected to one or more pulses of sufficient strength. The time for which stoppage occurs depends, essentially, on the magnitude of the pulses, the viscosity of the fluid and the radius of the pipe as well as on the frequency of the pulses.

\section{REFERENCE}

1. B. van der Pol and H. Bremmer, Operational calculus, Cambridge University Press, 1950.

\section{AN EXTENDED UPPER BOUND THEOREM ON THE ULTIMATE LOADS OF BUCKLED REDUNDANT TRUSSES ${ }^{1}$}

By E. F. MASUR (University of Michigan)

Introduction. This note is intended to supplement an earlier paper ${ }^{2}$ in which the collapse load of a buckled redundant rigid-jointed truss was bracketed between a lower and an upper bound. As was pointed out at that time, the lower bound theorem permits the computation of a sequence of "statically admissible" parameters which approach the

${ }^{1}$ Received August 22, 1955. This paper was written under the sponsorship of the Office of Ordnance Research, Contract No. DA-11-022-ORD-1156 while the writer was at Illinois Institute of Technology.

${ }^{2}$ E. F. Masur, Lower and upper bounds to the ultimate loads of buckled redundant trusses, Quart. Appl. Math. 11, 385-392 (1954). 